\title{
Markers of Autolysis in Acute ST Elevation Myocardial Infarction - A Comparative Analysis
}

\author{
Prabindra Maharjan, ${ }^{1}$ Reezu Manandhar, ${ }^{1}$ Wei Xu, ${ }^{1}$ Shuting Ma, ${ }^{1}$ Wenqi Han, ${ }^{1}$ Yan liu, ${ }^{1}$ \\ Yuan Zhou, ${ }^{1}$ Yue Wu, ${ }^{1}$ Caofeng Sun, ${ }^{1}$ Zuyi Yuan' \\ 'Department of Cardiovascular Unit, First Affiliated Hospital of Xi'an Jiao Tong University School of Medicine, Xi'an, \\ Shaanxi, China.
}

\begin{abstract}
Introduction: The availability of reliable noninvasive markers for infarct-related artery (IRA) patency status are very limited, otherwise could allow early identification of patients with patent IRA, for whom repeat thrombolysis or rescue percutaneous coronary intervention (PCI) are not necessary.

Methods: We conducted a single centered retrospective study of STEMI patients undergoing primary PCI to determine how various factors such as demographic characteristics, risk markers of coronary heart disease, clinical and blood parameters present differently in patients with higher coronary flow and patent infarct related artery from patients with total occlusion at the time of initial angiography and how they affect in outcome of the disease.
\end{abstract}

Results: MPV level (11.96 fL vs. 10.92 fL, P < 0.001), Lp (a) level (179.57 nmol/1 vs $141.16 \mathrm{nmol} / 1$, p < 0.001), CK-MB (290.2 vs. 190.98, P < 0.001), total cholesterol level (4.11 mmol/L vs. $3.8 \mathrm{mmol} / \mathrm{L}, \mathrm{p}$ $<0.02$ ) in total occlusion group were higher than in the patent IRA group. Wall motion abnormality was $77.2 \%$ for 203 patients with total occlusion group and $54.2 \%$ for 83 patients with patent IRA group $(\mathrm{P}<0.01)$. Mean hospital stay days were higher in total occlusion group as compared to the patent IRA group $\mathrm{P}<0.01$.

Conclusions: MVP, Lp (a), TC, and CK-MB levels and myocardial wall motion at the presentation may play the role of markers for IRA patency status that will help in early identification of patients with IRA, for whom repeat thrombolysis or rescue PCI may not be required.

Keywords: acute coronary syndrome; infarct related artery; myocardial infarction.

\section{INTRODUCTION}

Over seven million people die every year from coronary heart disease, accounting for $12.8 \%$ of all deaths. ${ }^{1}$ In China, Cardiovascular diseases (CVD) cause one third of all deaths, and the number is expected to be double by $2020^{2}$. Acute myocardial infarction (AMI) is caused by complex interaction among the atherosclerotic plaque, platelet activation, thrombus formation and coronary vasospasm. ${ }^{3}$ ST-segment elevation myocardial infarction (STEMI) signifies total occlusion of a coronary artery leading to myocardial necrosis. Approximately $10-20 \%$ of patients with acute STEMI presents with a patent infarct-related artery on the initial angiogram showed spontaneous coronary recanalization before angioplasty and the prognosis of these patients is significantly better. ${ }^{4}$ Although there are numerous research predicting different kinds of factors and its role in development of coronary artery disease (CAD), the study about factors that causes the autolysis of coronary thrombosis in patient with STEMI during its early stage, are very limited.

Correspondence: Prof. Zuyi Yuan, Department of Cardiovascular Unit, First Affiliated Hospital of $X_{i}{ }^{\prime}$ an Jiao Tong University School of Medicine, Xi'an, Shaanxi, China. Email : zuyiyuan@mail.xjtu.edu.cn, Phone : +8629-85323664. 


\section{METHODS}

We conducted a single centered retrospective study of STEMI patients undergoing primary $\mathrm{PCl}$ at the Department of Cardiology, $1^{\text {st }}$ Affiliated Hospital of $\mathrm{Xi}^{\prime}$ an Jiaotong University, from $1^{\text {st }}$ January 2008 to $31^{\text {st }}$ December 2013.

This study compiles with the declaration of Helsinki, and the research protocol was approved by the Ethics Commiittee of $\mathrm{Xi}^{\prime}$ an Jiao Tong University. Informed consents were also obtained from all study subjects as usual.

Total of 871 patients underwent primary $\mathrm{PCl}$ during this period, among whom 584 patients were excluded for following reasons: with documented history of coronary artery disease; development of accelerated idioventricular rhythm, a new bundle branch block; with history of thyroid related disorders, chronic systemic illness and other comorbid conditions; patients admitted to the hospital after 24 hours period from the onset of symptoms; patients undergoing thrombolysis before CAG.

Remaining 287 patients were then divided into two study groups on the basis of TIMI flow grade. Group A $(n=203)$, total occlusion group, included patients with TIMI flow $\leq 1$; group $B(n=83)$, patent IRA group, included those with TIMI flow $\geq 2$.

Data analysis was performed using SPSS 20 statistical package. Descriptive analysis of numerical data was expressed as mean, standard deviation and range, test of Normality was conducted within the groups. Variation between the two study groups were analyzed using $\mathrm{t}$ test or Mann Whitney $\mathrm{U}$ test depending upon the normality test. In case of categorical variables, they were expressed as frequency and percentage. Categorical data was analyzed using chi-square test. If the $P$ value was $<0.05$ then it was considered statistically significant.

\section{RESULTS}

\section{Patients}

Our study included 203 patients in total occlusion and 84 patients in IRA groups. Patient's descriptive characteristics are summarized in the Table 1 and 2 .

\section{Demographic Characteristics}

The proportion of male patient in total occlusion group was higher as compare to that in patent IRA group (88.2\% vs. $78.6 \%, P<0.05$, Table 1$)$, while the age and different occupations among two groups has no significant differences (Table 1 and 2).

\section{Blood Parameters}

MPV level in total occlusion group (11.96 fL) compared to that in patent IRA group (10.92 fL) was significantly higher $(P<0.001$, Table 1$)$. However, there was no significant difference in other blood parameters such as hemoglobin level, blood sugar level, platelets count, PT, APTT, WBC count, serum creatinine, cystatin $C$ and uric acid between the two study groups (Table 1). Similarly, serum electrolytes such as sodium, potassium, calcium and enzymes those are tested for the liver function like AST, ALT, serum albumin and total protein, all were not significant between two groups (Table1).

\section{Cardiac biomarkers}

CK-MB was significantly higher in total occlusion group as compared to the patent IRA group (290.2 vs. 190.98, $P<0.001$, Table 1) however, there was no significant difference found in other biomarker Troponin I between the two groups (14.62 vs. 12.66, $P=0.34$, Table 1).

\section{Inflammatory and lipid biomarkers}

Patients with total occlusion had higher Lp (a) levels as compared to that in patients with patent IRA (179.57 $\mathrm{nmol} / \mathrm{l}$ vs $141.16 \mathrm{nmol} / \mathrm{l}, P<0.001$, Table 1 and Fig. 1). Total cholesterol level in total occlusion group (4.11 $\mathrm{mmol} / \mathrm{L})$ was higher as compared to that in the patent IRA group $(3.8 \mathrm{mmol} / \mathrm{L})$, this difference was statistically significant $(P=0.02$, Table 1$)$.

hs-CRP, HDL levels were relatively higher in patent IRA group but were statistically non significance $(P>0.05$, Table 1).

LDL, TG and homocysteine levels were higher in total occlusion group but were statistically non significance $(P>0.05$, Table 1).

\section{Risk factors of CHD}

Prevalence of dyslipidemia was higher in total occlusion group $(14.3 \%)$ as compared to patent IRA group $(2.4 \%)$, which was, statistically significant $(P<0.01$, Table 1 and Fig. 1). Smoking, hypertension were seen relatively higher in total occlusion group in compare to IRA group while diabetes and positive family history were seen higher in IRA group than in total occlusion group ( Table 1 and Fig. 1).

Diabetes was found to be more prevalent in patent IRA group $(15.5 \%)$ than that in total occlusion group $(11.8 \%)$, but the difference was not significant statistically $(P$ value $=0.44$, Table 1 and Fig. 1$)$. Positive family history was seen more in patent IRA group $(8.3 \%)$, as compared to that in total occlusion group $(7.9 \%)$, but 
it was statistically non significance $(P=0.50$, Table 1 and Fig. 1).

\section{Echocardiography}

Mean LV inner diameter during diastole between total occlusion group (53.29 $\mathrm{mm})$ and patent IRA group (52.38 mm) was non-significant $(P=0.507$, Table 1). Prevalence of regional wall motion abnormality in echocardiogram was significantly higher in total occlusion group as compared to patent IRA group (77.2\% vs 54.2\%, $P<0.001$, Table 1 and Fig. 1). Ejection fraction in patent IRA $(53.79 \%)$ was relatively higher as compared to total occlusion group (52.03\%), but was not statistically significant $(P=0.16$, Table 1 and Fig. 1). Patients with total occlusion of infarct related coronary artery had longer hospital stay duration as compared to that of patients with patent IRA (13.67 days vs. 11.43 days, $P<0.01$, Table 1 and Fig. 1$)$.

\begin{tabular}{|c|c|c|c|}
\hline & Total occlusion & Patent IRA & $P$ value \\
\hline $\mathrm{N}$ & 203 & 84 & \\
\hline Age (years) & $57.59 \pm 10.97$ & $59 \pm 11.3$ & 0.664 \\
\hline \multicolumn{4}{|l|}{ Gender } \\
\hline Male & $179(88.2 \%)$ & $66(78.6 \%)$ & $0.03 *$ \\
\hline Female & $24(11.8)$ & $18(21.6 \%)$ & \\
\hline Smoking & $133(65.5 \%)$ & $52(61.9 \%)$ & 0.56 \\
\hline Hypertension & $83(40.9 \%)$ & $37(44 \%)$ & 0.34 \\
\hline Diabetes & $24(11.8 \%)$ & $13(15.5 \%)$ & 0.44 \\
\hline Dyslipidemia & $29(14.3 \%)$ & $2(2.4 \%)$ & $0.001 *$ \\
\hline Family history & $16(7.9 \%)$ & $7(8.3 \%)$ & 0.50 \\
\hline MPV & $11.96 \pm 1.23$ & $10.92 \pm 1.13$ & $0.00008^{*}$ \\
\hline APTT & $32.37 \pm 3.50$ & $39.01 \pm 3.61$ & 0.36 \\
\hline PT & $12.85 \pm 0.598$ & $13.2 \pm 0.398$ & 0.481 \\
\hline Uric acid $(\mu \mathrm{mol} / \mathrm{L})$ & $355.03 \pm 94.65$ & $282.84 \pm 68.96$ & 0.68 \\
\hline Cystin C (mg/L) & $0.92 \pm 0.2$ & $0.87 \pm 0.1$ & 0.19 \\
\hline Creatinine $(\mu \mathrm{mol} / \mathrm{L})$ & $90.57 \pm 13.55$ & $78.77 \pm 28.84$ & 0.17 \\
\hline AST (units/L) & $84.85 \pm 13.86$ & $90.61 \pm 20.1$ & 0.24 \\
\hline ALT (units/L) & $45.35 \pm 24.74$ & $49.51 \pm 31.51$ & 0.21 \\
\hline Total protein & $62.69 \pm 5.13$ & $66.10 \pm 7.48$ & 0.22 \\
\hline Serum albumin & $39.45 \pm 2.45$ & $39.1 \pm 3.85$ & 0.62 \\
\hline Sodium & $141.30 \pm 3.45$ & $139.54 \pm 1.37$ & 0.306 \\
\hline Potassium & $3.8 \pm 0.4$ & $4.03 \pm 0.26$ & 0.35 \\
\hline Calcium & $2.27 \pm 0.12$ & $2.3 \pm 0.38$ & 0.304 \\
\hline CK-MB & $290.20 \pm 108.28$ & $190.98 \pm 109.62$ & $0.00014^{*}$ \\
\hline Troponin I & $14.62 \pm 20.83$ & $12.66 \pm 16.09$ & 0.34 \\
\hline hs-CRP & $25.8 \pm 10.4$ & $30.35 \pm 14.6$ & 0.212 \\
\hline Homocysteine & $30.66 \pm 10.17$ & $27.06 \pm 10.17$ & 0.481 \\
\hline Lp(a) & $179.57 \pm 80.59$ & $141.16 \pm 49.24$ & $0.0003^{*}$ \\
\hline LDL & $2.46 \pm 0.78$ & $2.39 \pm 0.48$ & 0.507 \\
\hline HDL & $0.99 \pm 1.15$ & $1.11 \pm 0.40$ & 0.62 \\
\hline Total Cholesterol & $4.11 \pm 1.17$ & $3.8 \pm 0.57$ & $0.02 *$ \\
\hline TG & $2.02 \pm 1.01$ & $1.53 \pm 0.66$ & 0.787 \\
\hline $\mathrm{EF}$ (mean) (in \%) & $52.03 \pm 8.9$ & $53.79 \pm 9.2$ & 0.16 \\
\hline LVID(mean) (in mm) & $53.29 \pm 5.23$ & $52.38 \pm 5.51$ & 0.507 \\
\hline Wall motion abnormality & $156(77.2 \%)$ & $45(54.2 \%)$ & $0.00012^{*}$ \\
\hline Time (in hours) & $6.42 \pm 3.09$ & $6.97 \pm 3.27$ & 0.14 \\
\hline Hospital stay (in days) & $13.67 \pm 5.65$ & $11.43 \pm 3.6$ & $0.0001 *$ \\
\hline
\end{tabular}

Values are expressed as raw data and mean \pm SD

MPV mean platelet volume, APTT activated partial thromboplastin time, PT prothrombin time, AST Aspartate transaminase, $A L T$ alanine transaminase, $C K-M B$ creatine kinase-MB, $h s-C R P$ high sensitive $C$ - reactive protein, $L p(a)$ lipoprotein(a), $L D L$ low density lipoprotein, $H D L$ high density lipoprotein, $T G$ triglycerides, $E F$ ejection fraction, $\angle V I D$ left ventricular internal dimension.

${ }^{*} P<0.005$ compare between two groups 
Yuan et al. Markers of Autolysis in Acute ST Elevation Myocardial Infarction - a Comparative Analysis

\begin{tabular}{lllll}
\hline Table 2. Occupations of subjects. & & & & \\
\hline & Overall & Total occlusion & Patent IRA & P value \\
\hline Farmer & $97(33.8 \%)$ & $66(32.5 \%)$ & $31(36.9 \%)$ & \\
Military & $31(10.8 \%)$ & $21(10.3 \%)$ & $10(11.9 \%)$ & \\
Business/corporate & $8(2.8 \%)$ & $7(3.4 \%)$ & $1(1.2 \%)$ & 0.59 \\
Worker / labor & $34(11.8 \%)$ & $27(13.3 \%)$ & $7(8.3 \%)$ & \\
Retired & $87(30.3 \%)$ & $63(31 \%)$ & $24(28.6 \%)$ & \\
Others & $30(10.5 \%)$ & $19(9.4 \%)$ & $11(13.1 \%)$ & \\
\hline
\end{tabular}
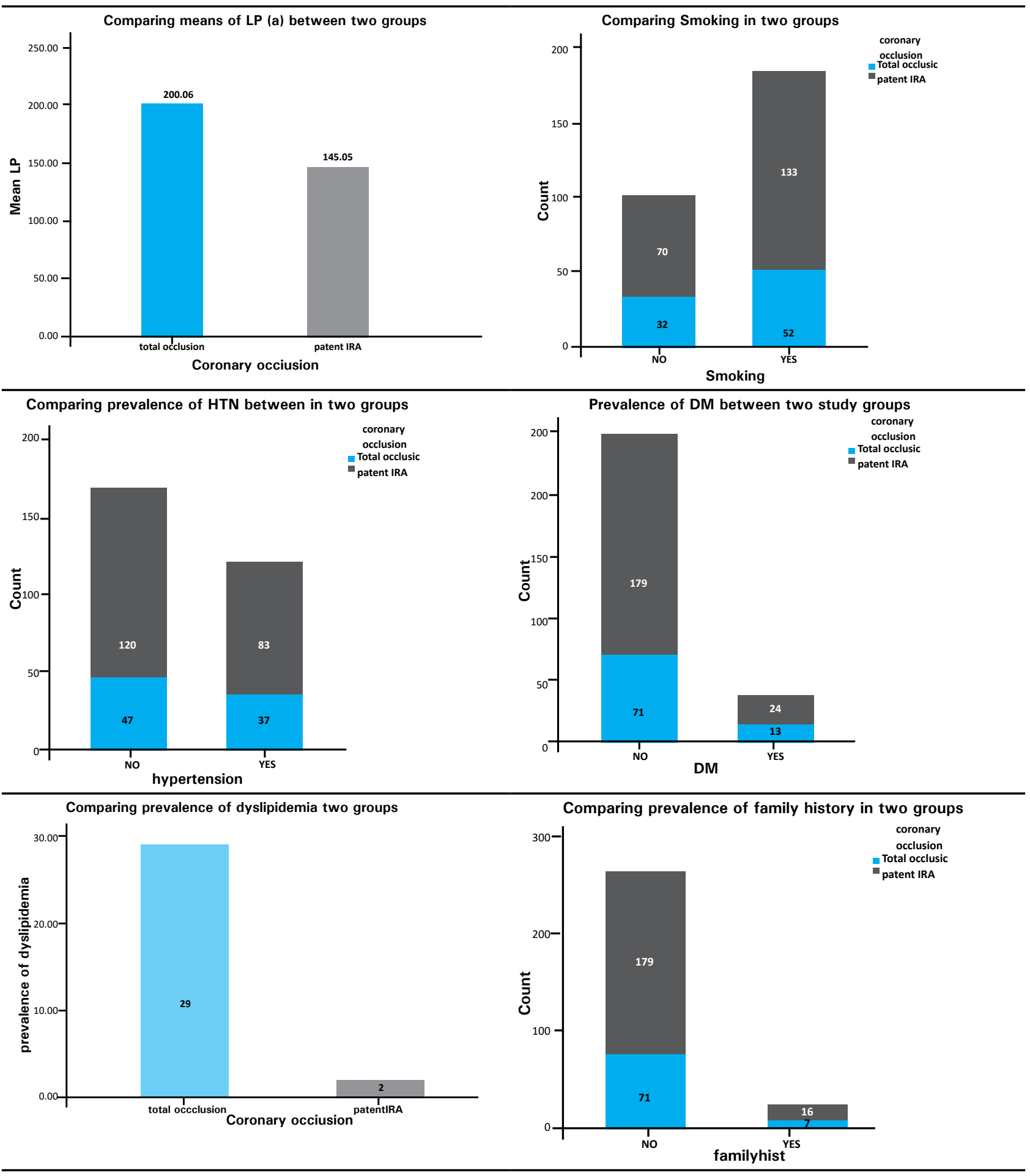

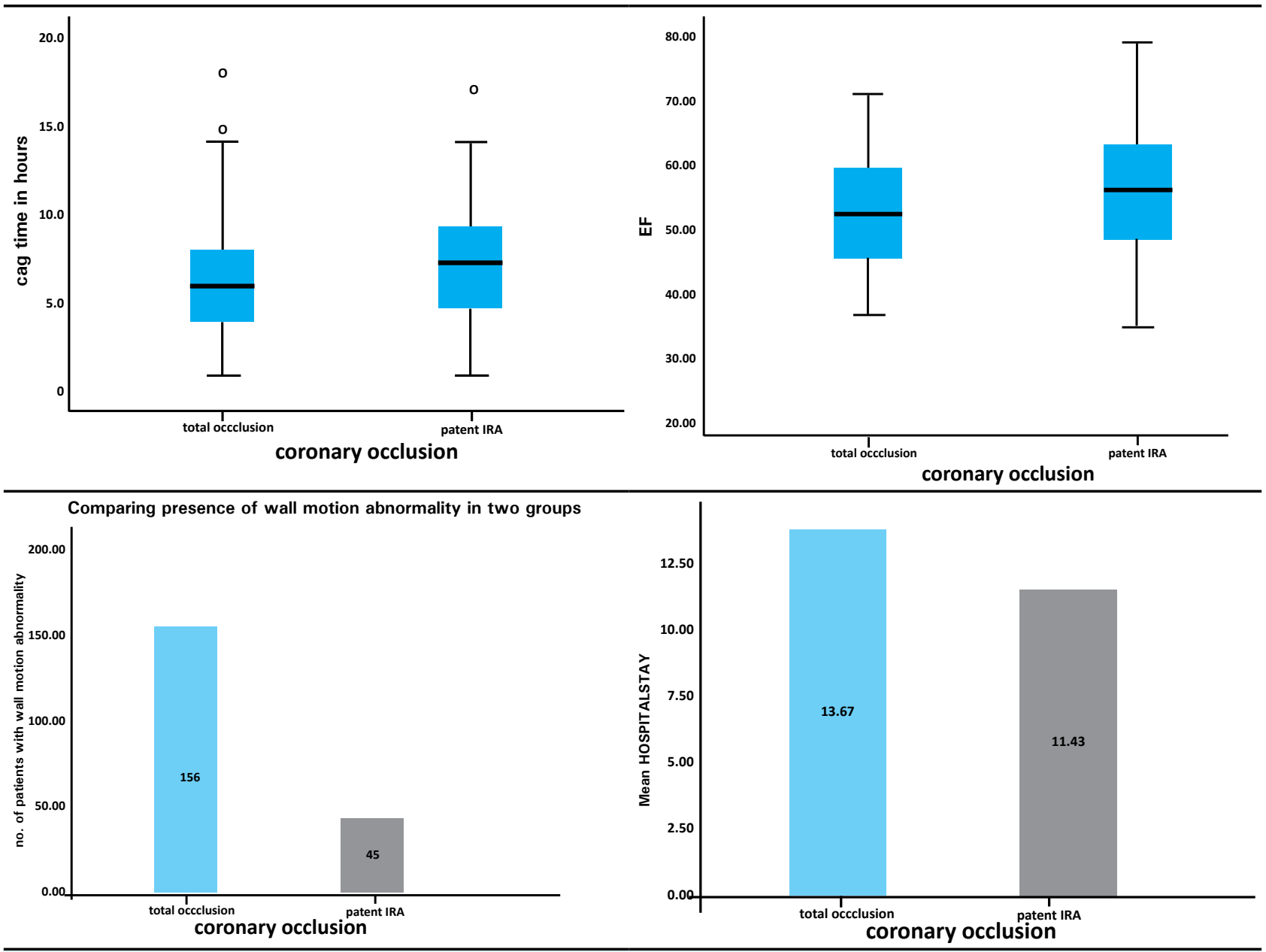

Figure 1. Descriptive characteristics of patients $L p(a)$ lipoprotein (a), Smoking, $H T N$ hypertension, DM diabetes mellitus, Dyslipidemia, family history, cag time coronary angiogram time, $E F$ ejection fraction, wall motion abnormality, mean hospital stay.

\section{DISCUSSION}

\section{Result Analysis}

Worldwide, coronary heart disease is leading cause of morbidity and mortality. STEMI, a more severe form of CHD, in which the coronary artery is totally occluded resulting in injury to the region of myocardium supplied by the particular epicardial artery, producing a characteristic changes on the ECG, the ST segment elevation. However, angiographic findings in all the patients with ST elevation MI do not have total occlusion of the infarct related artery. So to further clarify the main reasons as why some cases of ST elevation MI do not show total coronary occlusion, we compared the two groups of ST elevation MI undergoing emergency angiogram, divided on the basis of TIMI flow grade, one with total occlusion of infarct related artery whereas other group consisted of those with incomplete coronary occlusion. As TIMI flow grade one indicates total occlusion with minimal distal flow, we selected it as the cut-off point for division of two study groups and also, our study was based on ST elevation MI and total occlusion of the related artery, rather than post $\mathrm{PCl}$ tissue perfusion. ${ }^{5}$ The main aim of our study was to compare the two groups and analyze whether there exists any difference or it is the same and find out if any factor promote or prevent the autolysis of infarctrelated artery in ST elevation $\mathrm{MI}$, thus preventing total occlusion.

As we know older age is a very important marker for coronary artery disease ${ }^{6}$ and also the older patients had higher rates of TIMI flow grade of $0.7^{7}$ However, our study didn't show any significant difference between the two groups regarding the age of patients.

Male gender is considered as an independent risk factor for coronary heart disease. In our study, higher proportion of male patient had total occlusion as compared to their female counterpart, which was statistically significant. There was no any study which directly compared the gender variation in similar groups; however in a similar study, done by Julinda $\mathrm{M}$ et al., female gender was an independent predictor of higher rate of myocardial recovery and had lesser risk of perfusion defect. ${ }^{8}$ 
We also compared various blood parameters such hemoglobin level, blood sugar level, WBC, platelets count, PT and APTT but no any significant difference among the two study groups. However, mean of MPV in total occlusion group was higher than that in the patent IRA group with $P<0.05$. Platelets play an important role in the pathogenesis of coronary heart disease; MPV, a measure of platelet size, correlates with their reactivity. ${ }^{9,10}$ Orhan Maden et al. evaluated relationship of hematological indexes at the time of admission with myocardial reperfusion abnormalities in acute ST segment elevation myocardial infarction patients treated with primary percutaneous coronary interventions, in which, MPV was found to be independently associated with impaired reperfusion of infarct related artery ${ }^{11}$. This result was similar to that in our study, as MPV level was significantly higher in total occlusion group with $\mathrm{p}$ value less than 0.05 . Huczek $Z$ also reported that MPV on admission predicts impaired reperfusion and long-term mortality in acute myocardial infarction treated with primary $\mathrm{PCl}^{12}$ Result in this study was similar to that of our study, as it showed low MPV value was significantly associated with patent infarct related artery. Rodrigo Estévez-Loureiro et al. also reported that MPV predicts patency of the infarct-related artery in ST elevation $\mathrm{MI}$ before primary $\mathrm{PCl}$, as MPV was significantly higher in total occlusion group as compared to patent IRA group ( $\mathrm{p}$ value $<0.0001) .{ }^{13}$ Parameters related to kidney function such as serum creatinine, cystatin $C$ and uric acid were not significant between two groups implies their lesser role in autolysis of infarct related artery in ST elevation MI. Reduced renal function is shown by many studies to be associated with an increase in cardiovascular risk, as it prevalent in the elderly population. ${ }^{14}$ Serum creatinine was found to be an independent risk factor which significantly increased cardiovascular morbidity and mortality, when used as an indicator of impaired renal function in patients with existing CVD. ${ }^{15}$ Recently, cystatin C, a cysteine proteinase inhibitor, has been successfully used as an index of renal function along with creatinine. Some recent studies have indicated that cystatin $C$ is more superior indicator of cardiovascular events and mortality. ${ }^{16}$ As it is a marker of inflammation, hence leading to suggestion that it can be a risk factor for cardiovascular events. ${ }^{17}$ However, no studies were found which evaluated roles of creatinine or cystatin C in autolysis of infarct-related vessel of ST elevation MI.

Although cardiac biomarkers such as CK-MB and troponin I are used in the diagnosis and risk stratification of patients with suspected Coronary heart disease, it is not necessary for the patients who present with diagnostic ECGs of ST-segment elevation, as in these cases, $\mathrm{ACC} /$ American Heart Association (AHA) guidelines recommend immediate reperfusion therapy, without waiting for cardiac marker results ${ }^{18}$. However, recent studies have shown the usefulness of these biomarkers in predicting infarct-related artery patency, infarct size and LV function in patients with ST elevation MI. In our study, we evaluated these two commonly used markers between the two study groups. The mean of CK MB was significantly higher in total occlusion group as compared to the other group (with $p$ value less than 0.001 ); it signified that value of CKMB may correlate with degree of occlusion of infarct related artery in ST elevation MI. This result was similar to study done by Milenko J. Tanasijevic, et al. which also showed significant difference in median value of CK MB in patients with total occlusion of infarct related artery to that of patent IRA (TIMI flow grade 2 or 3), with $\mathrm{p}$ value $<0.001 .{ }^{19}$ They also showed there was no significant difference in median value of troponin I within six hours of onset of symptoms which equivocal with our result as $(P$ value $>0.05)$

Inflammatory markers and lipid parameters were also evaluated between our study groups. For most of the parameters such as hs-CRP, LDL, HDL, homocysteine and TG, the difference between the two study groups was statistically insignificant. A study done by Jin Won Kim et al. showed similar results in most of the parameters. It showed no significant difference in lipid parameters and hs-CRP between two study groups ( TIMI flow 0 or 1 and TIMI flow 2 or 3 ), as $\mathrm{p}$ value was more than 0.05 for HDL, LDL, TG and hs-CRP ${ }^{20}$. In our study, Lp(a) was shown to have significant effect on the patency of the infarct related artery, as there was statistically significant difference in the mean value of $L p(a)$ between the two study groups. This result was again similar to study done by Jin Won Kim. Similarly, according to Dagdelen's study the spontaneous reperfusion rate was significantly higher in patients with low levels of $L p(a)$ within first week of myocardial infarction. ${ }^{21}$ Another related parameter, serum cholesterol level was also found to be significantly higher in total occlusion group $(\mathrm{P}<0.05)$. There was no study supporting this result directly, however, different studies have reported that using cholesterol lowering drug have shown to enhance the patency of infarct related artery in myocardial infarction. ${ }^{22}$

Independent risk factors like smoking, hypertension, diabetes in our study were non significance $(P>0.05)$ between the two groups which was similar to the study done by Jin Won Kim, where he had compared between TIMI $\leq 1$ and TIMI $\geq 2$ groups. ${ }^{20}$ However, in our study, another risk factor, dyslipidemia, was higher in total occlusion group, which was statistically significant $(P<0.05)$. Meanwhile, like in the study by Rodrigo Estevez-Loureiro about effect of family history on coronary heart disease, we also didn't find significant difference between two groups $(P>0.05)$. There were 
no any studies showing positive family history being associated with the occlusion of infarct related artery in ST elevation MI patients.

Time from onset of symptoms to angiogram time was also evaluated between two study groups, which was statistically non-significant $(P>0.05)$. This result was similar to that from a study done by Jin Won Kim et al., in which, the time from symptoms to coronary angiogram was compared between different groups of TIMI flow and the difference was non-significant $(P>$ 0.05)..$^{20}$

We also evaluated findings from echocardiography between the two study groups. There was no significant difference between the two study groups for LVEF and LV diameter ( $P>0.05)$ similar to results of study done by Rodrigo Estévez-Loureiro et al. ${ }^{13}$ However, there was a significant statistical difference in occurrence of regional wall motion abnormality between the two groups $(P<0.05)$. Another parameter which is associated with the outcome of the disease is duration of hospital stay. When we evaluated the mean hospital stay duration in days between the two study groups, total occlusion group had higher mean hospital stay days than patent IRA group which was statistically significant $(P<0.05)$. There were no studies which directly compared the length of hospital stay in between the total occlusion and patent IRA group.

\section{Drawbacks, Limitations and Suggestions}

Main drawback of our study was that it was a retrospective study. So, the missing information about patients like their physical activities, dietary habit, height, weight was unable to recover. Also, about the details of patient's clinical features, for example, whether chest pain was relieved after taking sublingual nitrates at the time of presentation was unknown. This information was very important for evaluating the effect of coronary spasm and also could have been used for the preventive measures in the future. Another limitation of retrospective study was lack of proper history. For example, if patient was taking any medications such as aspirin, beta-blockers, nitrates, which might have a role in maintaining the patency of IRA, but didn't mention while recording the case history.

Another main drawback of our study was that, it was a single centered study. So diversity of the study population was limited. Different populations have different characteristic features, different lifestyles, different dietary habits and even different level of immune and inflammatory response.

So, a multi centered, prospective study is required to further analyze the markers affecting the patency of IRA before we can make any decision on its implication on the preventive and therapeutic measures.

\section{CONCLUSIONS}

Patients with lower levels of MPV, Lp(a), total cholesterol level and CK-MB at the time of presentation, without history of dyslipidemia, may have higher chance of having patent IRA in CAG in acute ST elevation Myocardial Infarction. The study also showed that patients with patent IRA may have lower chance of having myocardial wall motion abnormality and shorter duration of hospital stay.

Thus, MVP, Lp (a), TC, and CK-MB levels and myocardial wall motion at the presentation may play the role of markers for IRA patency status that will help in early identification of patients with IRA, for whom repeat thrombolysis or rescue $\mathrm{PCl}$ may not be required.

\section{ACKNOWLEDGEMENTS}

This study was supported by the National Basic Research Program of China ("973 Project" No. 2012CB517804 to Z. Yuan) and the National Science Fund of China for Distinguished Young Scholars (NSFC 81025002) to Z. Yuan. All procedures followed were in accordance with the ethical standards of the responsible committee on human experimentation (institutional and national) and with the Helsinki Declaration of 1975 , as revised in 2008 (5). Informed consent was obtained from all patients for being included in the study.

\section{REFERENCES}

1. World Health Organization Fact sheet N8310, updated June 2011. http://www.who.int/mediacentre/factsheets/fs310/ en/index.html

2. Xiao J-J, Chen Y-H. Prevalence of cardiovascular diseases in China[J]. Front Med China. 2010;4(1):16-20. doi:10.1007/ s11684-010-0007-8.
3. Widimsky P, Stellova B, Groch L, M Aschermann, M Branny, M Zelizko, et al. Prevalence of normal coronary angiography in the acute phase of suspected ST-elevation myocardial infarction: experience from the PRAGUE studies. Can J Cardiol 2006;22: 1147-52

4. Stone GW, Cox D, Garcia E, Brodie BR, Morice MC, Griffin $\mathrm{J}$, et al. Normal flow (TIMI-3) before mechanical reperfusion therapy is an independent determinant of survival in acute myocardial infarction: analysis from the primary angioplasty in myocardial infarction trials. Circulation 2001;104:636-41. 
5. Kern MJ, Moore JA, Aguirre F V, Bach RG, Caracciolo EA, Thomas Wolford, et al. Determination of Angiographic (TIMI Grade) Blood Flow by Intracoronary Doppler Flow Velocity During Acute Myocardial Infarction[J]. Circulation. 1996;94(7):1545-1552. doi:10.1161/01.CIR.94.7.1545.

6. Perk J, De Backer G, Gohlke H, Graham I, Reiner Z,Verschuren $\mathrm{M}$, et al. European Guidelines on cardiovascular disease prevention in clinical practice (version 2012) [J]. The Fifth Joint Task Force of the European Society of Cardiology and Other Societies on Cardiovascular Disease Prevention in Clinical Practice (constituted by re. Eur Heart J. 2012;33(13):1635-701. doi:10.1093/eurheartj/ehs092.

7. White HD, Barbash GI, Califf RM, Simes RJ, Granger CB, Weaver WD, et al. Age and Outcome With Contemporary Thrombolytic Therapy: Results From the GUSTO-I Trial[J]. Circulation. 1996;94(8):1826-1833. doi:10.1161/01. CIR.94.8.1826.

8. Mehilli J, Ndrepepa G, Kastrati A, Nekolla SG, Markwardt C, Bollwein $\mathrm{H}$, et al. Gender and myocardial salvage after reperfusion treatment in acute myocardial infarction[J]. J Am Coll Cardiol. 2005;45(6):828-31. doi:10.1016/j.jacc.2004.11.054.

9. Thompson CB, Jakubowski JA, Quinn PG, Deykin D, Valeri CR. Platelet size and age determine platelet function independently[J]. Blood. 1984;63(6):1372-5.

10. FrossardM,Fuchs I, LeitnerJM,HsiehK, VlcekM, LosertH, etal. Platelet function predicts myocardial damage in patients with acute myocardial infarction[J]. Circulation. 2004;110(11):13927. doi:10.1161/01.CIR.0000141575.92958.9C.

11. Maden O, Kacmaz F, Selcuk H, Selcuk MT, Aksu T, Tufekcioglu O, et al. Relationship of admission hematological indexes with myocardial reperfusion abnormalities in acute ST segment elevation myocardial infarction patients treated with primary percutaneous coronary interventions[J]. Can J Cardiol. 2009;25(6):e164-8.

12. Huczek Z, Kochman J, Filipiak KJ, Horszczaruk GJ, Grabowski M, Piatowski R, et al. Mean platelet volume on admission predicts impaired reperfusion and long-term mortality in acute myocardial infarction treated with primary percutaneous coronary intervention[J]. J Am Coll Cardiol. 2005;46(2):284-90. doi:10.1016/j.jacc.2005.03.065.

13. Estévez-Loureiro R, Salgado-Fernández J, Marzoa-Rivas R, Barge-Caballero E, Perez Perez A, Noriega-Concepsion $\mathrm{V}$, et al. Mean platelet volume predicts patency of the infarct-related artery before mechanical reperfusion and short-term mortality in patients with ST-segment elevation myocardial infarction undergoing primary percutaneous coronary intervention[J]. Thromb Res. 2009;124(5):536-40. doi:10.1016/j.thromres.2009.03.012.
14. Shlipak MG, Heidenreich PA, Noguchi H, Chertow GM, Browner WS, McClellan MB. Association of renal insufficiency with treatment and outcomes after myocardial infarction in elderly patients[J]. Ann Intern Med. 2002;137(7):555-62.

15. Shlipak MG, Simon JA, Grady D, Lin F, Wenger NK, Furberg CD. Renal insufficiency and cardiovascular events in postmenopausal women with coronary heart disease[J]. J Am Coll Cardiol. 2001;38(3):705-711. doi:10.1016/S07351097(01)01450-4.

16. Ix JH, Shlipak MG, Chertow GM, Whooley MA. Association of cystatin $\mathrm{C}$ with mortality, cardiovascular events, and incident heart failure among persons with coronary heart disease: data from the Heart and Soul Study[J]. Circulation. 2007;115(2):1739. doi:10.1161/CIRCULATIONAHA.106.644286.

17. Keller T, Messow CM, Lubos E, Nicaud V, Wild PS, Rupprecht $\mathrm{HJ}$, et al. Cystatin $\mathrm{C}$ and cardiovascular mortality in patients with coronary artery disease and normal or mildly reduced kidney function: results from the AtheroGene study[J]. Eur Heart J. 2009;30(3):314-20. doi:10.1093/eurheartj/ehn598.

18. Antman EM, Hand M, Armstrong PW, Bates ER, Green LA, Halasyamani LK, et al. 2007 Focused Update of the ACC/AHA 2004 Guidelines for the Management of Patients With ST-Elevation Myocardial Infarction: a report of the American College of Cardiology/American Heart Association Task Force on Practice Guidelines: developed in collaborat[J]. Circulation. 2008;117(2):296-329. doi:10.1161/ CIRCULATIONAHA.107.188209.

19. Tanasijevic MJ, Cannon CP, Antaman EM, Wybenga DR, Fischer GA, Grudzien C, et al. Myoglobin, CreatinineKinase-MB and Cardiac Troponin-I 60-Minute Ratios Predict Infarct-related Artery Patency After Thrombolysis for Acute Myocardial Infarction[J]. J Am Coll Cardiol. 1999;34(3).

20. Kim JW, Seo HS, Suh SY, Choi CU, Kim EH, Rha SW, et al. Relationship between lipoprotein(a) and spontaneous recanalization of infarct-related arteries in the early phase of acute myocardial infarction[J]. Clin Cardiol. 2008;31(5):211-6. doi:10.1002/clc.20143.

21. Dagdelen S, Eren N, Akdemir I, Karabulut H, Ergelen M, Caglar N. The effect of lipoprotein-a on thrombolytic therapy and spontaneous reperfusion in acute myocardial infarction. A comparative study [J]. Int Angiol. 2002 Dec;21(4):384-9.

22. Takayama M, Munakata K, Tomita Y, Kaneko H, Kunmi S, Yokoyama H, et al. 1006-45 Regression of Infarct-Related Coronary Lesions on Aggressive Lipid Lowering Treatment in Patients After Recovery from Acute Myocardial Infarction[J]. J Am Coll Cardiol. 1995;25(2):357A. doi:10.1016/07351097(95)92939-3. 